\title{
On Archimedes' statics
}

\author{
(Sobre la estática de Arquímedes)
}

\author{
Mario Bacelar VALENTE*
}

\begin{abstract}
Archimedes' statics is considered as an example of ancient Greek applied mathematics; it is even seen as the beginning of mechanics. Wilbur Knorr made the case regarding this work, as other works by him or other mathematicians from ancient Greece, that it lacks references to the physical phenomena it is supposed to address. According to Knorr, this is understandable if we consider the propositions of the treatise in terms of purely mathematical elaborations suggested by quantitative aspects of the phenomena. In this paper, we challenge Knorr's view, and address propositions of Archimedes' statics in their relation to physical phenomena.
\end{abstract}

KEYWORDS: statics, Archimedes, principle of the lever, mechanics.

RESUMEN: La estática de Arquímedes se considera como un ejemplo de las matemáticas aplicadas de la antigua Grecia; incluso es vista como el inicio de la mecánica. Wilbur Knorr defendió con respecto a este trabajo, como otros trabajos de él u otros matemáticos de la antigua Grecia, que carece de referencias a los fenómenos físicos que se supone que debe abordar. Según Knorr, esto es comprensible si consideramos las proposiciones del tratado en términos de elaboraciones puramente matemáticas sugeridas por los aspectos cuantitativos de los fenómenos. En este trabajo, desafiamos la idea de Knorr y abordamos las proposiciones de la estática de Arquímedes en su relación con los fenómenos físicos.

PALABRAS CLAVE: estática, Arquimedes, principio de la palanca, mecánica.

* Correspondence to: Mario Bacelar Valente. Departamento de Sistemas Físicos, Químicos y Naturales, Universidad Pablo de Olavide, ctra. de Utrera, km 1 (41013, Sevilla-España) - mar.bacelar@gmail.com - https://orcid.org/0000-0001-9473-5005

How to cite: Valente, Mario Bacelar (2020). «On Archimedes' statics»; Theoria. An International Journal for Theory, History and Foundations of Science, 35(2), 235-242. (https://doi.org/10.1387/theoria.20482).

Received: 23 December, 2018; Final version: 09 September, 2019.

ISSN 0495-4548 - eISSN 2171-679X / (C) 2020 UPV/EHU 


\section{Elements of Archimedes'statics}

The only extant mathematical treatment of the lever by Archimedes is the treatise Planes in Equilibrium. ${ }^{1}$ This work has a twofold purpose. On one side we have a mathematical approach to the statics of the lever; and on the other side we find the mathematical determination of centres of gravity. The term "planes" in the title is to be understood as making reference to plane figures; i.e., to geometrical figures, as described, e.g., in the part of Euclid's Elements addressing planar geometry (Euclid, 1908). The two issues addressed in the treatise are not however independent themes; simply, Archimedes devotes part of the work to the determination of the centres of gravity of different geometrical figures, which is not strictly necessary to his treatment of the statics of the lever. Thus, it is clear that the work also aims to address the determination of centres of gravity and goes beyond the consideration of the statics of the lever. ${ }^{2}$

Archimedes' treatise begins with seven postulates or principles. From these, and mathematical results demonstrated in other works, a series of propositions are demonstrated. Some of the principles are as follows:

1. Equal weights at equal distances are in equilibrium, and equal weights at unequal distances are not in equilibrium but incline towards the weight which is at the greater distance.

4. When equal and similar plane figures coincide if applied to one another, their centres of gravity similarly coincide.

6. If magnitudes at certain distances be in equilibrium, (other) magnitudes equal to them will also be in equilibrium at the same distance. (Heath, 1897, pp. 189-190; see also Mugler, 1971, p. 80)

We can notice that while there are references to geometrical terms, and implicitly to mathematical magnitudes (as we will see), there are terms which clearly are not mathematical. ${ }^{3}$ The terms "equilibrium" and "incline" make reference to physical phenomena related to levers. As stated in principle 1, interpreted as an experimental principle, if we suspend equal weights from the arms of a lever at an equal distance from the fulcrum of the lever, it will not move-it will be in equilibrium. If the weights are suspended at unequal distances, we observe that the arms of the lever move from the horizontal position towards the weight at a greater distance from the fulcrum. Principle 1, like the others, can be seen as general principles, gathered and corroborated by experience, the majority of which relate to a simple machine-the lever. Regarding "weight" and "distance", these are addressed in the treatise in two related ways. As expressing physical phenomena, principle 1 relates to weight

1 According to Heath, several treatises by Archimedes on mechanics might have been lost (Heath, 1921, pp. 28-9).

2 The rationale for this might well be the intention to supersede a previous erroneous method for the determination of a centre of gravity (see Dijksterhuis, 1956, pp. 303-4).

3 In this work, we adopt the term mathematical magnitude when referring to magnitudes as developed in the book 5 of Euclid's Elements, in relation to which one has the notions of ratio and proportional magnitudes. We can notice in principle 6 the term "magnitude". Here, it has a completely different meaning from "magnitude" in the Elements; it is used to denote a material body whose shape is not relevant, only its weight and centre of gravity. 
and distance as measurable quantities; however, as a principle of applied mathematics, it takes them as mathematical magnitudes. When observing the particular phenomena related to the lever leading to principle 1, its expression in terms of this principle only makes sense if we start from a practical notion of distance and weight. That is, we need to measure the weights and conclude that they are, in practical terms, equal, and the same for the distances. There is no difficulty with this. Both weight and distance (length) were determinable in ancient Greece in terms of metrological systems. We have units of weight in relation to which we determine the weight of a body. Equivalently, we have units of length in relation to which we can determine distances. ${ }^{4}$ Having measured two bodies as being, in practical terms, of the same weight, and suspending them from each arm of a lever at distances measured as the same, we observe that the lever maintains its equilibrium-the arms do not move and are still on the horizontal. This is the phenomenon that is expressed in the first part of principle $1 .^{5}$

However, in relation to the role of principle 1, or others, in the demonstrations of the various propositions, the reference to weight and distance cannot be read as referring to metrological notions-weight and distance as measured in relation to some adopted units. Weight and distance are treated (represented) as mathematical magnitudes. This will become clear when addressing the propositions. In this way, while we might consider at first principle 1 , like the others, in relation to the phenomena it describes, as an experimental principle, as applied in the treatise it must be understood as a principle of applied mathematics. A principle that while retaining references to the description of physical phenomena related to levers-and terms like "equilibrium" and "incline" are only meaningful in this context-, treats "metrological" concepts like "weight" and "distance" using notions from pure mathematics-weight as a mathematical magnitude and distance as the mathematical magnitude of a straight line.

Another crucial notion for Archimedes' statics is that of centre of gravity. It is referred to in a few of the principles but, like with other terms, there is no explicit definition of it. However, important aspects of the notion of centre of gravity are implicit in the treatise. As Eduard Dijksterhuis mentioned, principle 6 can be understood as stating that the equilibrium of a lever is not affected if the bodies that are suspended from their centre of gravity are substituted by other bodies with different shapes but with the same weight as that of each of the original bodies and suspended also from their centres of gravity (Dijksterhuis, 1956, p. 293). It is as if in what regards the effect of the weight of bodies on the equilibrium of the lever, all of a body's weight is "concentrated" at the centre of gravity. In Archimedes' statics, the centre of gravity is given a mathematical description. More exactly, it is represented as a geometrical point. For example, proposition 4 states the following:

If two equal magnitudes have not the same centre of gravity, the centre of gravity of the magnitude composed of the two magnitudes will be the middle point of the straight line joining the centres of gravity of the magnitudes. (Dijksterhuis, 1956, p. 288)

4 On ancient Greek metrology see, e.g., Gyllenbok (2018).

5 As mentioned by a reviewer: "the principle implicitly assumes indifference to other differences-not merely to differences of color and material, but to position right and left of the fulcrum". 
Regarding the bodies suspended from a lever, these are represented as planar geometrical figures. For example, principle 4 explicitly refers to planar figures (as does the title of the treatise). Principles 1,2, 3, and 6 are general in the sense of making a mathematical rendering of the description of physical phenomena related to a lever with bodies of different shapes suspended from it. Principles 4, 5, and 7 do not have that generality. We can see them as stating results related to thin bodies (Heath, 1897, pp. 189-190; Mugler, 1971, pp. $77 \& 80-1)$.

Another geometrical representation at play is that of the lever itself. The lever is represented as a straight line (and its fulcrum as a point). This can be seen, e.g., in proposition 3, in which the straight line, joining two unequal weights $\mathrm{A}$ and $\mathrm{B}$, represents a lever, and a point $\mathrm{C}$ of this straight line represents its fulcrum. The lever is in equilibrium for some distances $\mathrm{AC}$ and $\mathrm{BC}$ of the centres of gravity of the weights (points $\mathrm{A}$ and $\mathrm{B}$ ) from the point C. Proposition 3 states that unequal weights can only be in equilibrium when the greater weight is at a lesser distance from the fulcrum than the other weight. That is, AC must be less than BC (Heath, 1897, pp. 190-1).

The early propositions of the treatise (one to five) can be seen as preparatory or even necessary to the demonstrations of propositions 6 and 7, which state that two magnitudes (bodies) are in equilibrium at distances reciprocally proportional to their weights. This result is known as the law or principle of the lever (see, e.g., Renn et al., 2003, p. 48; Dugas, 1957, p. 24). Take A to be the weight of the first body described as a mathematical magnitude. Likewise, $B$ is the weight, as a mathematical magnitude, of the second body. $E$ and $\mathrm{D}$ are the centres of gravity of the first and second body respectively. They are points on a straight line representing a lever. Finally, let $\mathrm{C}$ be the point on the straight line representing the fulcrum of the lever. EC is the distance (as a mathematical magnitude) of the centre of gravity of body A from the point C, and DC is the distance of the centre of gravity of body $\mathrm{B}$ from point $\mathrm{C}$. The demonstrations of the propositions show that the ratio of the mathematical magnitudes $\mathrm{A}$ and $\mathrm{B}$ is the same as the ratio of the mathematical magnitudes DC and EC (Heath, 1897, pp. 192-4; see also Mugler, 1971, pp. 85-8). That is, the magnitudes are in the same ratio, in the mathematical sense of book 5 of Euclid's Elements. ${ }^{6}$

While we are considering propositions of applied mathematics, we can note, as was the case with other propositions, that the main statement of the propositions is made in mathematical terms. That is, the phrase "two magnitudes are in equilibrium at distances reciprocally proportional to their weights" must be interpreted as meaning, using a modern standard notation, A:B::DC:EC (see, e.g., Netz, 2004, p. 5). As previously mentioned, the principles are already couched in mathematical terms, as can be seen when taking them into account in the demonstration of the propositions. For example, in proposition 3 we consider principle 1 in relation to two possibilities, that of the mathematical magnitudes $\mathrm{AC}$ and $\mathrm{BC}$ being equal or AC being greater than BC (Heath, 1897, pp. 190-1). Principle 1 can only be meaningfully applied in the demonstrations of propositions if the reference to distances in it is to distances as mathematically described, and not as measured distances. However, being the principles a mathematical rendering of the description of

6 In Euclid's Elements, the ratios are always of magnitudes of the same type. The adoption of ratios between different types of magnitudes is a much later development. Possibly, its earliest relevant application was made by Galileo (see, e.g., Valente, 2019). 
physical phenomena related to levers or centres of gravity, and relying on terms like "equilibrium", "incline", or "centre of gravity", they are not principles of pure mathematics. Should we expect a deduction from these principles - a proposition-to be also about the phenomena taken into account in these principles? But how to address, in these propositions (or in their demonstrations), the simultaneous reference to terms related to the description of the phenomena and to mathematical terms?

What is at issue is the status of Archimedes' propositions in relation to pure mathematics and to physical phenomena. Clarifying this might help to better understand Archimedes' applied mathematics in the case of statics - to understand his propositions about statics and the interplay of the references to physical phenomena and to pure mathematics.

\section{Knorr's thesis and our challenge to it with respect to Archimedes' statics}

That Archimedes' treatise is one of applied mathematics seems quite uncontroversial. In fact, Archimedes' statics is seen as establishing the foundations of a new exact sciencethe science of mechanics, which, nowadays, is one of the branches of physics (Dugas, 1957, p. 24; Mugler, 1971, p. 77). However, when considering a view proposed by Knorr, we might question what being applied mathematics in the case of Archimedes' statics might mean and imply. Regarding Archimedes' treatise, and other works by him and other authors, Knorr asks the following: "If they indeed sought knowledge of the physical phenomena, why should they conceal the links between their theorems and the phenomena?" (Knorr, 1989, p. 323). Knorr answer his question with the following thesis:

If we view the present set of treatises not as efforts toward the mathematical analysis of certain classes of physical phenomena, but instead as the elaboration of fields in pure geometry suggested by the quantitative relations applying to these phenomena, then we obtain a different perspective. The suppression of phenomenal references would be bizarre in a theory devoted to the explanation of those phenomena; but it is entirely appropriate in a purely mathematical inquiry into the geometric relations they satisfy. (Knorr, 1989, pp. 324-5)

That the principles are principles of applied mathematics should not be controversial. They are based on physical phenomena related to the lever, but they include the mathematical rendering of parts of the description of the phenomena. For example, as mentioned, principle 1 treats "weight" and "distance" - that in the practice related to the phenomena under consideration are metrologically defined-as mathematical magnitudes. However, we do not agree with Knorr when he writes that in his treatise Archimedes "suppresses overt mechanical references, as to weights or balances, and speaks instead in terms of magnitudes and equilibrium" (Knorr, 1989, p. 323): Weights are mentioned throughout the work when needed (but treated as mathematical magnitudes); magnitudes (in the principles and propositions) refer to material bodies considered only with respect to their weights and centres of gravity, not to the mathematical magnitude; balances or levers are represented by straight lines; "equilibrium" is not a mathematical term (using Knorr's phrasing, it is a mechanical term).

While in this work Archimedes does not give a definition of centre of gravity, as mentioned, principle 6 refers to an important feature of the centre of gravity: when a body is suspended from its centre of gravity it is as if all its weight is located there. As Knorr ac- 
knowledges, Archimedes had a "physical conception" of centre of gravity which he mentioned in his treatise Quadrature of the parabola (Knorr, 1989, p. 318). What the phrasing "physical conception" means is that Archimedes had what we might call an experimental knowledge of how to determine the centre of gravity of a material body. According to Archimedes: "Every suspended body, no matter what its point of suspension, assumes an equilibrium state when the point of suspension and the centre of gravity are on the same vertical line" (cited in Assis, 2010, p. 124; see also Mugler, 1971, p. 171). As Andre Assis mentions, this statement regarding the centre of gravity shows that Archimedes knew a practical procedure to determine the centre of gravity (Assis, 2010, p. 124).

That Archimedes' physical conceptions on statics go beyond what we might consider being present in the treatise's principles (or left implicit), does not have to bear on Knorr's views. As Knorr mentioned, Archimedes' treatise “does not necessarily represent the complete range of [his] views on the nature of mechanics" (Knorr, 1989, p. 318). However, the principle of the lever demonstrated in his work, in our view, shows that Archimedes had a previous knowledge of this principle as what we have called an experimental principle-i.e. a principle describing physical phenomena. As mentioned, the first part of the treatise is a sequence of demonstrations of propositions leading to the demonstration of the principle of the lever. Should we suppose that Archimedes chose his principles in some ad hoc way and then by mere luck found that proposition 6 and 7 could be deduced from part of them? We think this simply cannot be the case. The principle of the lever was already known previous to Archimedes. In the treatise called Mechanical problems, the law or principle of the lever had already been formulated and addressed, even if in terms different from those of Archimedes (Renn et al., 2003, pp. 46-7). Accordingly, "the law must have been well known when it was given a proof a generation or two later by Euclid and Archimedes" (Renn et al., 2003, p. 47). Importantly, the formulation of the principle of the lever did not happen ex nibilo, it arose in the context of "the practical knowledge related to the balance with unequal arms" (Renn et al., 2003, p. 47).

We could imagine a treatise alternative to that of Archimedes in which the principle of the lever is taken as one of the principles, and from it and other principles some propositions are derived. As such its status must be the same as that of the principles of the treatise of Archimedes. Simply, Archimedes organized his work in such a way that this principle was derived-as a proposition-from other "simpler" principles. Being possibly a very wellknown description of a physical phenomenon related to the lever (as its presence in the earlier Mechanical problems leads us to think), it would not make sense to Archimedes to press the point that it refers to physical phenomena. This would be self-evident. Contrary to Knorr's view, there is no suppression of phenomenal references. Also, in relation to the sequence of demonstrations ending with propositions 6 and 7, we do not assist to the elaboration of statics in terms of pure geometry, what we have is the deduction of an experimental principle-rendered as an applied mathematics proposition-from other experimental principles that are rendered as applied mathematics principles. ${ }^{7}$

It is a fact that in his treatise Archimedes also demonstrates a series of propositions related to the centre of gravity. The motivation for this might be, as mentioned, the intention to supersede a previous erroneous method for the determination of a centre of gravity. But even if this is so, the sequence of demonstrations in book 2 leading to proposition 8 where Archimedes determines the centre of gravity of a segment of a parabola, relies on a highly sophisticated mathematical approach. Here, we might 
Propositions 6 and 7, like, e.g., principle 1, must be understood in the context of a previous understanding of phenomena associated to levers. In particular, "weight" and "distance" are, first of all, to be considered in terms of metrological systems. We can consider the description of physical phenomena, which are rendered in part mathematically in principle 1 and propositions 6 and 7, without reference to pure mathematics. As it is the statement of these experimental principles can be the same as that in applied mathematics:

Experimental principle 1: Equal weights at equal distances are in equilibrium, and equal weights at unequal distances are not in equilibrium but incline towards the weight which is at the greater distance.

Experimental principle of the lever: Two magnitudes (bodies) are in equilibrium at distances reciprocally proportional to their weights.

Here, we simply consider "weight" and "distance" in terms of measures attained adopting the corresponding metrological systems. In this context, of measurements, the terms "equal", "unequal", or "proportional" must not be understood as terms of pure mathematics, but, e.g., in terms of practical measurements and the practical equality or inequality of measured weights and distances (or the practical reciprocal proportionality of the division of the weights to the division of the distances).

In Archimedes' applied mathematics these experimental principles are in part rendered mathematically. For example, weights are described as mathematical magnitudes; the same happens with distances between two points on a straight line. This will enable, in propositions 6 and 7, to relate the ratio of two weights (which is meaningful because weights are treated as mathematical magnitudes) to the rate of two distances (also treated as mathematical magnitudes). We obtain a pure mathematics result: one ratio is proportional to the other. However, we still make reference, e.g., to terms like "equilibrium" and "incline" in the demonstrations. In this way, while, e.g., the final result of the demonstration of propositions 6 and 7, corresponds to what we now might call a mathematical expression (A:B::DC:EC), the mathematical terms are a representation of terms adopted in the description of physical phenomena related to levers. In particular, "weight" as a mathematical magnitude is a representation of "weight" as determined in terms of a metrological system of units (to simplify, a weight as measured in practice). The same applies, mutatis mutandis, to distances. To further extract the practical meaning of the applied mathematics proposition, one only needs to take into account that the straight line going from $\mathrm{D}$ to $\mathrm{E}$ represents the lever, and $\mathrm{C}$ its fulcrum. The mathematical result translated into an experimental principle means that when two unequal weight (as measured) are suspended from the arms of a lever, the ratio (the division) of the practically measured distances (as numbers) must be reciprocally proportional to the ratio (the division) of the practically measured weights (as numbers). Contrary to Knorr's view, we do not have a development of statics in pure geometry.

Ultimately what Archimedes achieves in this part of his statics is to show that the experimental principle of the lever can be deduced from other experimental principles. In the

seem to have a vindication of Knorr's position - an elaboration of statics in terms of pure geometry. But there is an important difference. Even in the case of the centre of gravity of a segment of a parabola, Archimedes is not actually making an elaboration of the field in pure geometry. Archimedes is working in applied mathematics, not pure mathematics. 
process he makes explicit that pure mathematics can be used to represent part of the physical phenomena in a way that is integrated with the non-mathematical description of other aspects of the physical phenomena (e.g. when using the notions of equilibrium and inclination), such that from experimental principles (rendered as applied mathematical ones) one can deduce applied mathematics propositions that are the rendering of other experimental principles.

\section{Acknowledgements}

I would like to thank the comments and suggestions of the anonymous reviewers.

\section{REFERENCES}

Assis, A. (2010). Archimedes, the center of gravity, and the first law of mechanics, second edition. Montreal: Apeiron.

Dijksterhuis, E. (1956). Archimedes. Copenhagen: Ejnar Munksgaard.

Dugas, R. (1957). A history of mechanics. London: Routledge \& Kegan Paul Ltd.

Euclid (1908). The thirteen Books of the Elements, second edition. Translated with introduction and commentary by Sir Thomas L. Heath, from the critical edition of Heiberg. Cambridge: University Press.

Gyllenbok, J. (2018). Encyclopaedia of historical metrology, weights, and measures, vol. 1. Cham: Birkhäuser.

Heath, T. (1897). The works of Archimedes. Cambridge: University Press.

Heath, T. (1921 [1981]). A history of Greek mathematics, vol. 2. New York: Dover Publications.

Knorr, W. (1989). The practical element in ancient exact sciences. Synthese 81, 313-328.

Mugler, C. (1971). Archimède, vol. 2. Paris: Les Belles Lettres.

Netz, R. (2004). The works of Archimedes, vol. 1. Cambridge: Cambridge University Press.

Renn, J., Damerow, P., and McLaughlin, P. (2003). Aristotle, Archimedes, Euclid and the origin of mechanics: the perspective of historical epistemology, in J. L. Montesinos Sirera (ed.), Symposium Arquimedes. Fundación Canaria Orotava de Historia de la Ciencia. Preprint 239. Max-Planck-Institut für Wissenschaftsgeschichte.

Valente, Mario B. (2019). Geometry of motion: some elements of its historical development. Artefactos. Revista de estudios sobre la ciencia y la tecnología 8(2), 5-26.

Mario Bacelar Valente currently develops his research based at the University Pablo de Olavide. He has a European $\mathrm{PhD}$ in the philosophy of physics by the University of Seville. His research interests include different sciences. For the time being, his output has focused on history and philosophy of physics and mathematics.

Address: Departamento de Sistemas Físicos, Químicos y Naturales, Universidad Pablo de Olavide, Ctra. de Utrera, km 1, 41013, Sevilla, España. E-mail: mar.bacelar@gmail.com 